\title{
Challenging the paradigm
}
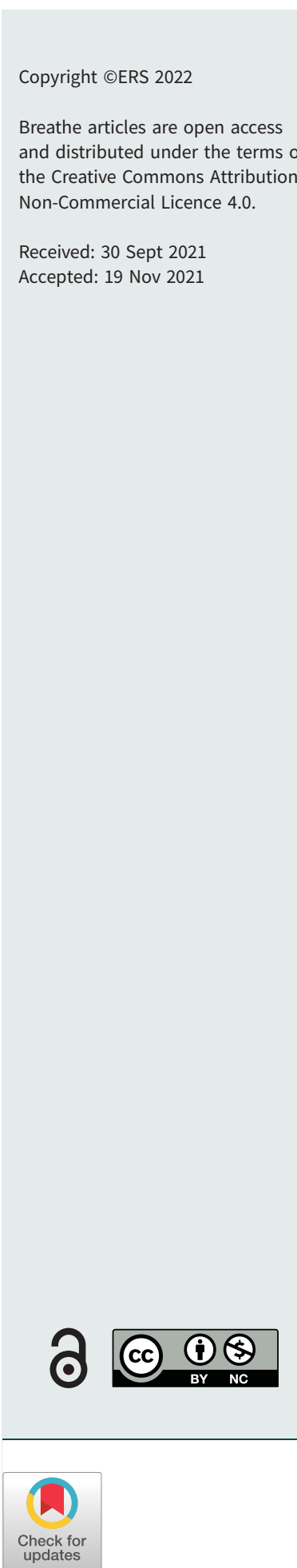

\section{To the Editor:}

I read with interest the review by Bush and PAVORD [1] regarding the suggestion that we should abandon "umbrella" diagnoses and rather we should address "treatable traits" when looking after patients with airways disease. Unfortunately, aspects of their proposal are only likely to add to, rather than reduce, the current confusion regarding management of these conditions. More importantly, it seems to include some potentially very dangerous recommendations.

There have been several publications over the past 6 years suggesting that there might be improved outcomes in airways disease by addressing so-called "treatable traits". Most have simply advocated good clinical practice as was outlined to me as a medical student 40 years ago. Any clinician wishing to provide high-quality holistic patient care should be considering factors such as comorbidities, poor compliance, mental health issues and lifestyle issues, including smoking, and indeed such recommendations are contained in most guidelines. The third educational aim touches on this but the article fails to provide any detail.

However, this review goes a step further suggesting abandoning "umbrella terms" and simply treating "traits", with eosinophilic inflammation being their focus. While this might appear to sidestep the need to understand the underlying nature of particular diseases and the need to consider that there may be more than one active process contributing to a patient's symptoms it does not take us forward and is potentially dangerous. The author's model would suggest that a patient with proven $\beta$-agonist bronchodilator responsiveness should be treated with a short- (SABA) and/or long-acting $\beta$-agonist (LABA), but that if the same individual does not have evidence of eosinophilic airways disease inhaled corticosteroids (ICS) should be withheld (page 5), a suggestion that would fly in the face of all current guidelines. This proposal seems to be predicated on a simplistic belief that corticosteroids simply address eosinophilic airways inflammation. However, corticosteroids have been shown to have numerous actions not least being their ability to significantly reduce airways smooth muscle (ASM) hypersensitivity, modify ASM function and cytokine release [2, 3]. These effects are observed in both atopic and non-atopic asthma patients [2, 3]. Given that the majority of asthma patients (those who have proven lability in ASM length [3]), do not demonstrate airways eosinophilia and that there are no studies that demonstrate that corticosteroids are ineffective in this population this proposal is potentially very harmful. A logical conclusion of this line of argument is that prednisolone should be withheld from those hospitalised with a significant exacerbation of asthma if they have not been shown to have had eosinophilic inflammation. Moreover, it is almost universally believed that LABAs should not be prescribed without ICS because they have been shown to be associated with excess deaths in at least some populations when used as monotherapy, while one of the authors has argued that SABAs should not be prescribed to most asthmatic patients because of the dangers they pose [4]. Their argument is further undermined by their comment that airways eosinophilia is not stable over time and by the observation that monitoring sputum eosinophilia does not result in improved outcomes in children with asthma [5].

Two of the papers cited are used to support the suggestion that corticosteroids are only effective in eosinophilic airways inflammation. While Harry Morrow Brown's contributions to the field have indeed been outstanding, the paper they refer to regarding prednisolone [6] was an open study of patients with doctor diagnosed asthma who were apparently experiencing ongoing bronchoconstriction despite $\beta$-agonist therapy. Lung function was not performed, nor was there any indication of the patients ages. He commented on the "grave difficulty" he had in distinguishing those with chronic bronchitis and those with asthma especially in older patients and noted that those with the former condition who were included in

Shareable abstract (@ERSpublications)

While advocating for addressing "treatable traits" is admirable in that it reminds clinicians to consider the patient and not the disease, the use of this idea to promote dangerous changes in practice should be challenged. https://bit.ly/30VkP8Q

Cite this article as: Everard ML. Challenging the paradigm. Breathe 2022; 18: 210148 [DOI: 10.1183/ 20734735.0148-2021]. 
the study, unsurprisingly, did not respond to prednisolone. He also concluded that prednisolone "never does more than suppress the asthmatic state and it does not abolish eosinophils in the sputum". Further he noted eosinophils could be present in asymptomatic subjects and that the deciding factor (for starting prednisolone therapy) was "the severity of the bronchospasm and not (sic) the findings in the sputum". There is no comment about neutrophil numbers in the sputum.

The current review also suggested that "some children and adults have a pauci-inflammatory picture, and do not respond to ICS” [1]. However, the reference they provide [7] does not support this suggestion given that it is a review of non-eosinophilic asthma that argues that the majority of asthma patients are not atopic and do not have eosinophilic airways inflammation. Rather it supports the suggestion that eosinophilic inflammation is neither necessary nor sufficient for the development of asthma [3]. No one is arguing that eosinophilic inflammation (most commonly associated with allergy) does not result in the release mediators that can induce airways narrowing in those with asthma (with its loss of ASM homeostasis) and exacerbate the condition, but they are not a core feature of asthma.

In their obsession with eosinophils the authors omit to discuss the chronic dysbiosis of the microbiome of the conducting airways (chronic bacterial bronchitis) that is responsible for so much respiratory morbidity and indeed is probably the cause of much of the fixed airways obstruction they refer to. However, the failure of the respiratory fraternity to effectively deal with this issue (with or without asthma) is one of a number of "elephants in the room" when it comes to airways disease. At present we do not have a clear approach to diagnosis and more importantly, to effective treatment; simply ignoring it as being too hard to address does not help. Paediatric experience with early aggressive treatment suggests more proactive, aggressive, and early treatment represents good practice amongst paediatric patients [8] and this is probably true for adults [9]. Neutrophils maybe in the airways for numerous reasons including a bacterial dysbiosis, acute respiratory viral infections [10], inhalation of pollutants and/or cigarette smoke, corticosteroid therapy, and probably, through stressing ASM cells and epithelial cells as airways constrict. As with eosinophils they do not define asthma any more than a chronic airways dysbiosis is the defining feature of cystic fibrosis.

The most successful approach to dealing with asthma has been the Finnish programme based on a clear diagnosis with objective evidence of ASM responsiveness, early use of ICS and early intervention during exacerbations [3, 11]. Despite the enormous efforts to produce and promote guidelines in the UK, the healthcare system continues to fail huge numbers of patients with asthma and other airways diseases. Good practice normally involves doing the simple things well and rather than inventing new paradigms we should be advocating for an evidence-based structured approach based on proven success, such as the Finnish model. Addressing "treatable traits", when the term refers to the promotion of sound medical practice addressing the whole patient, should be encouraged. However devising paradigms that do not advance the field and that, if taken to their logical conclusions, are potentially dangerous should not go unchallenged.

Asthma is a discrete condition (though when used loosely the term unfortunately becomes an umbrella term) characterised by loss of the normal homeostatic mechanisms that prevent excessive narrowing [3]. There is no doubt that for some asthmatic patients eosinophilic inflammation helps drive symptoms, but eosinophils are neither necessary nor sufficient to cause asthma. The suggestion that withholding steroids from the majority in whom eosinophilic inflammation does not appear to be a driver of symptoms is very dangerous.

\section{Mark L. Everard}

Paediatric Respiratory Medicine, University of Western Australia, Perth, Australia.

Corresponding author: Mark L. Everard (mark.everard@uwa.edu.au)

Conflict of interest: None declared.

\section{References}

1 Bush A, Pavord ID. Challenging the paradigm: moving from umbrella labels to treatable traits in airway disease. Breathe 2021; 17: 210053. 
2 Bel EH, Timmers MC, Hermans J, et al. The long-term effects of nedocromil sodium and beclomethasone dipropionate on bronchial responsiveness to methacholine in nonatopic asthmatic subjects. Am Rev Respir Dis 1990; 141: 21-28.

3 Anthracopoulos M, Everard ML. Asthma: a loss of post-natal homestatic control of airways smooth muscle with regression towards a pre-natal state. Front Pediatr 2020; 8: 95.

4 Bush A. Preventing asthma deaths: above all, do no harm. Lancet Respir Med 2019; 7: 732-733.

5 Fleming L, Wilson N, Regamey N, et al. Use of sputum eosinophil counts to guide management in children with severe asthma. Thorax 2012; 67: 193-198.

6 Brown HM. Treatment of chronic asthma with prednisolone; significance of eosinophils in the sputum. Lancet 1958; 2: 1245-1247.

7 Douwes J, Gibson P, Pekkanen J, et al. Non-eosinophilic asthma: importance and possible mechanisms. Thorax 2002; 57: 643-648.

8 Ishak A, Everard ML. Persistent and recurrent bacterial bronchitis - a paradigm shift in our understanding of chronic respiratory disease. Front Pediatr 2017; 5: 19.

9 Martin MJ, Lee H, Clayton C, et al. Idiopathic chronic productive cough and response to open-label macrolide therapy: an observational study. Respirology 2019; 24: 558-565.

10 Duros K, Everard ML. Time to say goodbye to bronchiolitis, viral wheeze, reactive airways disease, wheeze bronchitis and all that. Front Pediatr 2020; 8: 218.

11 Haahtela T, Tuomisto LE, Pietinalho A, et al. A 10 year asthma programme in Finland: major change for the better. Thorax 2006; 61: 663-670. 